\title{
The Influence of the Philosophical Concept of Yin and Yang on the Modeling of Folk Patterns
}

\author{
Hong Nie \\ Art Design Department \\ Wuhan University of Science and Technology \\ Wuhan, China
}

\author{
Sibo Yang \\ Art Design Department \\ Wuhan University of Science and Technology \\ Wuhan, China
}

\begin{abstract}
Throughout history, the Chinese concept of Yin and Yang has a long history and is deeply rooted. The philosophy of Yin and Yang includes the idea of Yin-Yang and eight diagrams, the concept of "Yin and Yang interact and generate all things" which centers on the reproduction worship, and the philosophical concept of Yin-Yang and Five Elements. These traditional ideas not only run through the system of folk arts modeling, but also have a profound impact on the folk culture. We need to understand the philosophy contained in these ideas and make these philosophical concepts guide the creation and development of modeling in future.
\end{abstract}

Keywords-folk patterns; the philosophical concept of YinYang and eight diagrams; the philosophical concept of "Yin and Yang interact and generate all things"; the philosophical concept of Yin-Yang and Five Elements

\section{ON THE YIN-YANG PHILOSOPHY}

Yin and Yang are the most fundamental concepts in the ancient Chinese philosophy, which originally refer to the positive and negative faces of the sun - the face toward the sun is Yang, and the back face is Yin; they are always presented by the climate. Through long-term observation, the ancient thinkers pointed out that all things have positive and negative aspects; they also summarized the opposition and succession of nature as the concept of Yin and Yang. For example, Bo Yangfu in Western Zhou Dynasty mentioned the concept of Yin-Yang coordination and affirmed that the contradictory forces of Yin and Yang are inherent in things. ${ }^{1}$

After many years of experience and research, the ancient people concluded that the Two Forms are Yin and Yang, heaven and earth, which are represented by "-" and "--" in the symbols of trigrams; the four symbols are Taiyin, Taiyang, Shaoyin and Shaoyang, which represent four seasons (spring, summer, autumn and winter) and four directions (north, south, east and west); the Eight Diagrams are Qian, Kun, Zhen, Xun, Can, Li, Gen and Dui, which symbolize heaven, earth, thunder, wind, water, fire, mountain and lake and are always called Jing diagrams - if two of the eight diagrams are arranged as a group, the hexagrams are formed2. This is a classification of time, space and all things. Fuxi drew the eight diagrams, which means that he created a series of symbolic systems through studying the above knowledge. In Book of History: Hong Fan, the traditional philosophers put forward the idea of
"Five Elements" for the first time, which fully illustrates that the most basic substances constitute the world as indispensable elements, namely water, fire, wood, gold and soil $^{3}$.The philosophical system of Yin-Yang, Eight Diagrams and Five Elements runs through the entire Chinese traditional philosophy and actually embodies the thought of "unity of heaven and humanity ". The so-called eight diagrams are a kind of embodiment and generalization of nature and human society as well as a summary of everything in the world. This is also a kind of traditional philosophy that was born in a chaotic and differentiated state and shows the continuous reproduction and flourishing of all creatures.

It is the traditional modeling and fine arts that reflect this philosophical concept in the earliest days. The idea of Yin and Yang not only plays a core role in the eight diagrams of Yin and Yang, but also has left an indelible imprint on the Chinese art history; meanwhile, the philosophical concept of the interaction of Yin and Yang as the source of all things and the concept of Yin-Yang and Five Elements have a farreaching influence on the folk patterns as in "Fig. 1".
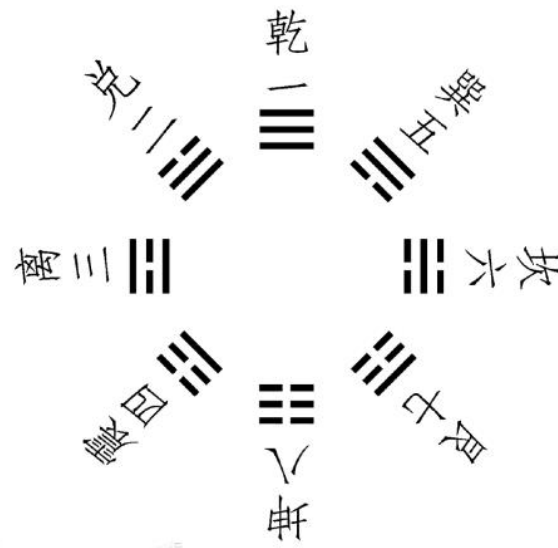

Fig. 1. Fuxi drew the eight diagrams. 


\section{APPLICATION OF THE YIN-YANG AND EIGHT DIAGRAMS IN FOLK PATTERNS}

\section{A. Concept of Yin-Yang and Eight Diagrams}

The eight diagrams are also known as Jing diagrams, which refer to the eight basic diagrams in Zhouyi. The eight diagrams derive from the divination of the primitive religions. The picture of eight diagrams is composed of Tai ji Diagram and the symbols of eight diagrams, which has become a special symbol of rich national cultural connotations. The idea of "Tai Ji and eight diagrams" is a summary and application of the traditional thought of Yin-Yang and Five Elements, and it concentratedly reflects people's reverence for nature, such as their worship for earth, mountains, wind, water and dragons, as well as many taboos included in this worship(such as time, directions, places, etc.). In Tai Ji Diagram, the pattern is like two lively fish in spirit: they chase and attract each other and thus form a circular. Therefore, the traditional philosophers jokingly called this pattern Yin-Yang fish. In the paper-cut work "Two Fish and Eight Diagrams", the pattern of eight diagrams is surrounded by eight groups of auspicious patterns, such as flowers, plants, butterflies, etc.

\section{B. Expression of the Philosophical Concept of "the Integration of Yin and Yang Produces all Things" in Folk}

The philosophers believe that the eight diagrams can be matched repeatedly to show the good or ill luck of one thing or even of the future. For example, in the eight diagrams, it is widely believed that things can be promising and auspicious only through changing and developing upward; stagnation without changes means that things are unpromising and inauspicious. Because the heaven is Yang-heavy and the earth is Yin-heavy, Yin and Yang float toward the opposite direction; hence, heaven and earth can interact and cause violent changes. This is called auspicious divination. On the contrary, as for the so-called inauspicious divination, heaven is above and earth is below, and they cannot produce intersection, so the changes will not take place. Because there is no future, it is an inauspicious divination6. This theory is usually used in the folk patterns of the family residences. For example, on the door of Miao people in west Hunan, we can often see wooden Tai Ji and eight diagrams, which mean stabilizing home and driving away evil spirits. In the famous pattern "Mirror of Eight Diagrams", the blackand-white Yin-Yang diagram is surrounded by the embroidered sunlight, which means "the sun is shining brightly from all directions"; at the same time, this typically embodies the concept of "the integration of Yin and Yang produces all things"; in addition, we can see the five poisonous creatures embroidered near the main pattern, which mean mutual generation and restriction at the same time. Through small patterns, the picture of Yin-Yang eight diagrams reflects the ancient people's grand view of spacetime and world, and it also shows people's concept of fertility worship which is characterized by mutual interaction, continuous reproduction and mutual restraint as shown in "Fig. 2" and "Fig. 3".

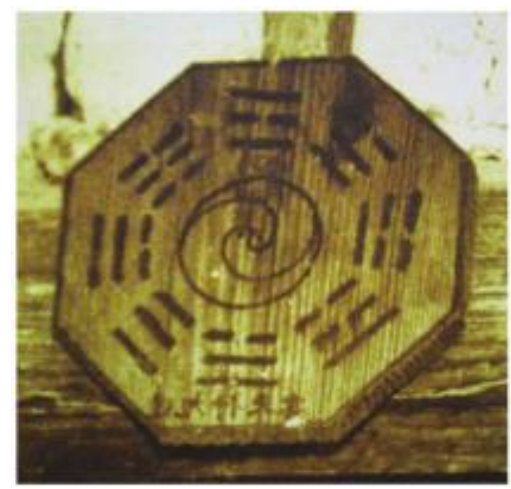

Fig. 2. Wooden Tai Ji and eight diagrams.

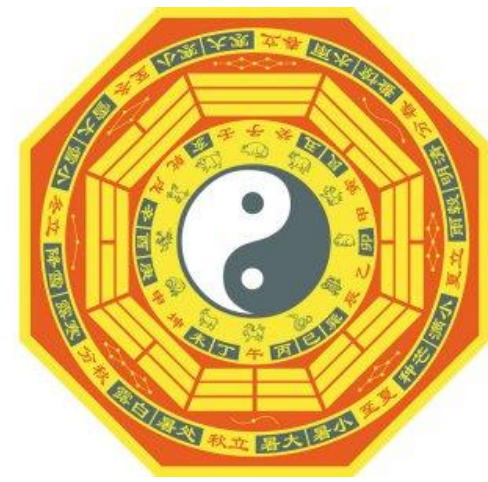

Fig. 3. Mirror of Eight Diagrams.

\section{Application of the Pattern of Yin-Yang and Eight Diagrams in the Modeling of Folk Patterns}

In the folk opera, there are Tai Ji and eight diagrams on the masks and the costumes of Emperor of Huang and Lord Lao $\mathrm{Zi}$ of the Great Monad. The famous paper-cut "Unity of Taoism and Buddhism" is derived from the concept of YinYang and Five Elements, among which "Buddhism" (Fo) is a homophone for "bat" in Chinese. "Taoism" (Tao) is formed by the pattern of two fish that are revolving and interact with each other, and four patterns of water wave are used to represent the meaning of the eight diagrams, as if this is to tell people that Taoism and Buddhism have returned to their original nature through these elements4.In addition, The Tai Ji and eight diagrams are also used in China's residential buildings, such as the gilt camphor wood plaque with eight diagrams of Qing Dynasty. In Chinese people's mind, Yin and Yang are not static concepts but two dynamic elements that people recognize from the eternal movement of life. According to the intelligent Chinese people, the schema of "binary opposition" is inextricably linked with "Tai Ji" and "Taoism". If we say that there is a concept of "eternal spirit structure" of natural color in the ordinary Yin-Yang pattern, the pattern of Yin-Yang and eight diagrams has already become an "eternal cultural and mental structure" in the Chinese civilization as shown in "Fig. 4" and "Fig. 5". 5 


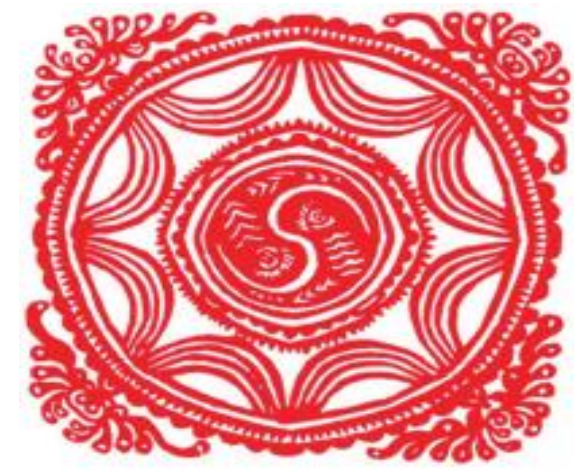

Fig. 4. The paper-cut "Unity of Taoism and Buddhism".

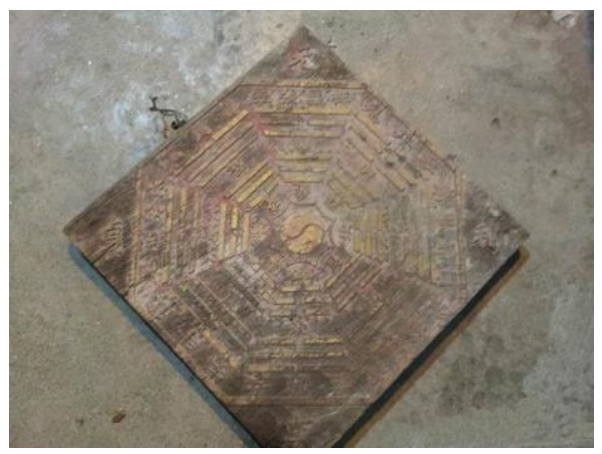

Fig. 5. The gilt camphor wood plaque with eight diagrams of Qing Dynasty.

\section{THE INFLUENCE OF THE PHILOSOPHICAL CONCEPT OF "YIN AND YANG INTERACT AND GENERATE ALL THINGS" ON FOLK PATTERNS}

\section{A. Chinese People'S Traditional Concept of Reproduction}

The traditional Yin-Yang philosophy originates from people's reverence for reproduction. In Yi Zhuan, "When men mate with women, all creatures are generated" refers to "the heaven consists of Yin and Yang, the changes of which are called Tao" and "men have the character of Qian Tao and women have the character of Kun Tao; the interaction between them can generate all things." Wang Anshi said: "everything has a mate." In the Chinese folk, there is an old saying that "good things come in pairs". Therefore, in Chinese people's concept of life reproduction, there is a strong sense of even number. As a result of this, in the patterns of folk arts, the works of fine arts combining the idea of unity of opposites with the traditional concept of reproduction emerge in an endless stream.

\section{B. The Mode of "Yin-Yang Fish" in Folk Patterns}

The essence of Chinese people's philosophy system and art system is the unity of heaven and man and the identity of object and self. For the creators of the folk fine arts in China, fish is a culture symbol of the reproduction of human beings and all creatures, which can be realized only through the mate of Yin and Yang fish. For example, when the folk artists present fish — the actual objects, they prefer to express their feelings by using things, while the material aspect is described slightly and vaguely, which shows their wish to make use of objects to express ideas. In the eyes of folk artists, fish is a special kind of symbol representing people's reverence and worship for reproduction in the concept of Yin and Yang. People think that the mate of two fish is a reflection of reproduction, which embodies that they have endowed "fish" with philosophical meaning in the art forms of their works. The endless succession of generations can be ensured only by reproduction, and people think that "rotation" is a special kind of reproduction; therefore, many art works usually take "rotation" as core idea. For example, some people draw the symmetric and rotary fish and use this special movement to present the organic interaction of Yin and Yang in the universe, so that the reproduction of human beings and all creatures can be continued. Drawing two fish in the symbolic plate of the matrix of universe in the opposite direction refers to the philosophical concept and symbolic code that Yin and Yang are contained in the matrix of the chaotic universe and their interaction can generate human beings and all creatures and ensure the continuous production of them as shown in "Fig. 6".

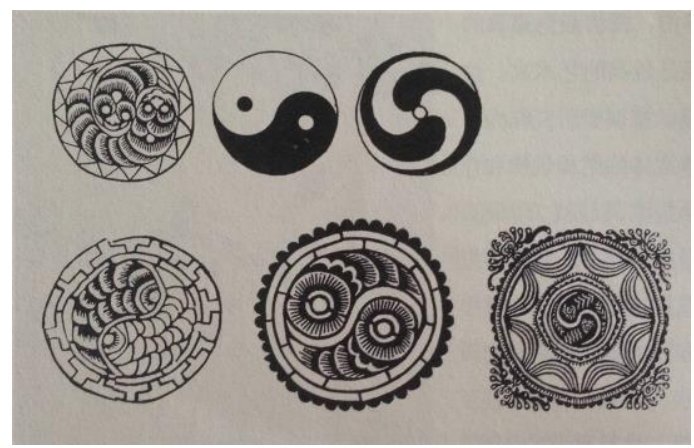

Fig. 6. Yin-Yang fish.

\section{The Unified and Opposite Idea of "Yin and Yang Interact and Generate all Things" Influences the Modeling of Folk Patterns}

In folk, there is a pattern called "clasped bowls", which refers to a kind of bowl with strange shape. The bowl above represents heaven and the bowl below stands for earth; hence the idea of "Yang above and Yin below" is reflected in the "clasped bowls" which represent a chaotic universe where Yin and Yang are mixed --- they can be divided into two or combined into one. In the folk art works, there are also a pattern that the clasped bowls are opened and surrounded by Yin-Yang fish, which means that the Yin-Yang principle can generate all creatures. The character of "double happiness" can also reflect the ancient people's grand view of universe and primitive sexual consciousness. Through the character of "double happiness", we can see that many folk art works always maintain the value system that Yin and Yang are integrated and Tao produces all creatures. It can be seen that the familiar patterns in the folk arts are not the simple phenomenon of "even number" and the embodiment of "compound image"; they are also the concentrated expression of the primitive culture in philosophical ideas. These patterns representing the unity and opposition of Yin and Yang are all derived from the philosophical concept of 
"Tao engenders one, one engenders two, two generates three, and three generates all creatures" as shown in Fig. 7 and "Fig. $8 "$.

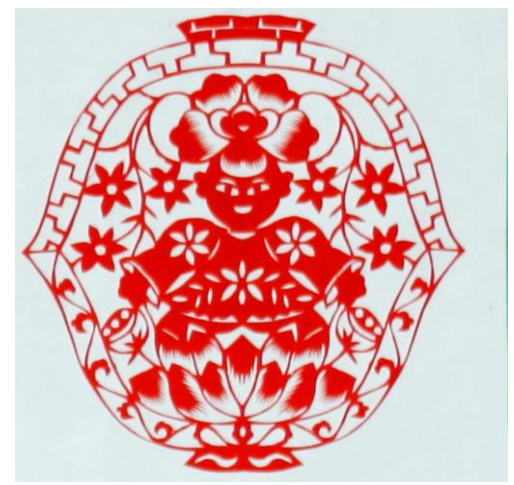

Fig. 7. The paper-cut "clasped bowls".

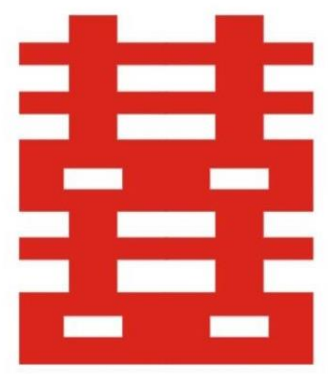

Fig. 8. The paper-cut "double happiness".

\section{The Profound Influence of the Philosophical Concept of Yin-Yang and Five Elements on Folk Patterns}

1) The philosophical concept of Yin-Yang and Five Elements: The idea of unity of opposites in Yin-Yang and Five Elements makes the Chinese traditional art form more colorful. The ancient philosophers classified all things through the five elements' material, shape, nature, function and color, and the characteristics of the five elements were abstracted. The patterns created by artists all have their own attributes of Yin-Yang and Five Elements; besides, the design of these patterns contains the idea of the balance of Yin and Yang and the mutual generation and restriction of the five elements, which enters into the mysterious realm of "unity of heaven and man" and follows the tenet of "all figures should have meanings, and the meanings should be auspicious". Here the five elements refer to the five basic substances — gold, wood, water, fire and soil. Through long-term practice in life, the ancient people in China realized that gold, wood, water, fire and soil are all indispensable substances that constitute the universe; on this basis, they developed an idea that all things in the world are generated by the motion and change of the five basic substances. These substances generate and restrain each other in a constant change, and they maintain a dynamic balance in the continuous motion of mutual generation and restriction.

2) The guiding role of the space concept in Yin-Yang and Five Elements for the modeling creation of folk patterns: With the in-depth study of the ancient Tai Ji in China, the ancient people profoundly realized the universe, time and space. Accordingly, in the folk arts, they showed their understanding of the concepts characterized by the unity of opposites, such as perfection, symmetry, dynamic-and-static, black-and-white, etc. In creating the folk patterns, the Chinese folk artists did not follow the western principle of perspective; instead, they adopted the idea of modeling in the Chinese philosophical concept of origin. For example, in the paper-cut work "The grand gathering of Spring Festival" of Zhongyang in Shanxi, when the scene of celebrating the grand gathering of Spring Festival is showed in the central courtyard of the quadrangle dwellings, the whole scene is completely displayed without the shading of each plane. The houses in the four corners of the courtyard fall down, with their bottoms toward the center. The people celebrating the grand gathering also fall down, with their feet toward the center, which reflects the space concept of combination of the five elements in China's philosophy of origin. This modeling method that breaks the spatio-temporal norm seems so harmonious and unified only when it appears in the folk arts as shown in "Fig. 9" and "Fig. 10".

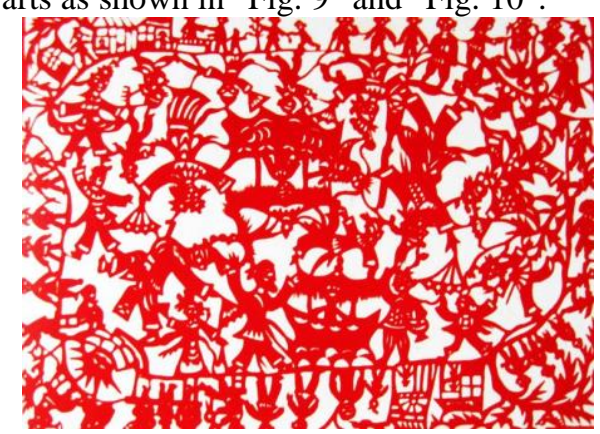

Fig. 9. The paper-cut work "The grand gathering of Spring Festival" of Zhongyang in Shanxi figure.

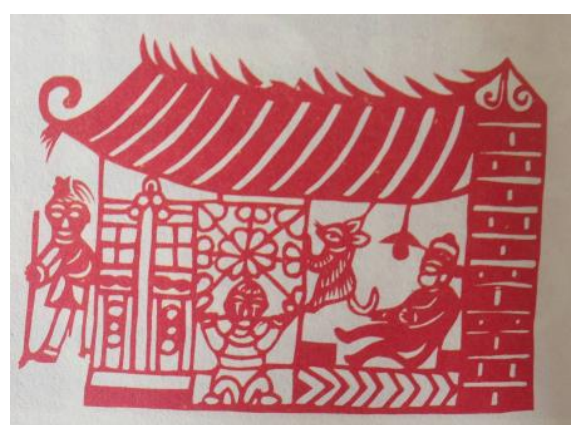

Fig. 10. The folk paper-cut work "Farm Life" of northern Shaanxi.

3) "Yin-Yang and Five Elements" in folk patterns: In the Chinese folk arts, the five elements are also the fundamental basis of the folk art patterns, such as the personified masks containing the five elements. At the same time, the idea of the five elements influences the basic coloring of the folk art. 
For example, in the folk woodblock New Year pictures, five colors are often used, including the saturated primary colors such as red, yellow and green, as well as black and white. The various colors and the five elements have corresponding symbolic meanings. Green in the east represents wood, white in the west represents gold, red in the south represents fire, black in the north represents water, and yellow in the middle represents soil. With regard to the masks for the folk grand gathering of Spring Festival and the masks for Di Opera and Nuo Opera, the five colors are also marks for judging the characters, and there is a principle that "red means loyalty and braveness while white means treacherousness, black means honesty and uprightness while gray means courage, yellow means violence while blue means outlaws, and green means warriors while pink means the ages". In China, the folk watermark woodblock New Year pictures are also printed with five colors. These colors are bright with high saturation; if we stick these pictures on the walls, the room will become bright. All kinds of folk patterns that are based on the five colors have distinct features, and the magic charm of the five colors is fully displayed.

In short, the idea of the unity of opposites of Yin-Yang and Five Elements has given a unique style to the creation of the folk art, and it also makes the folk art more brilliant as shown in "Fig. 11" and "Figure. 12".

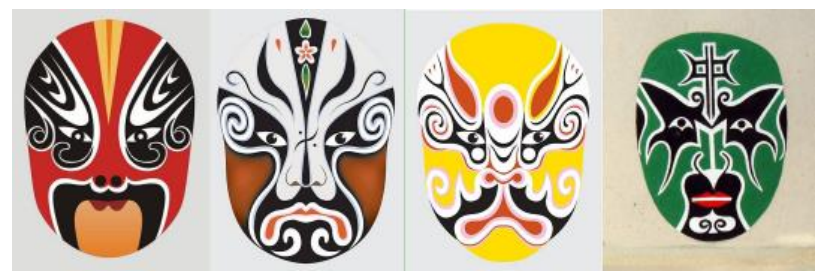

Fig. 11. The masks for Peking Opera.

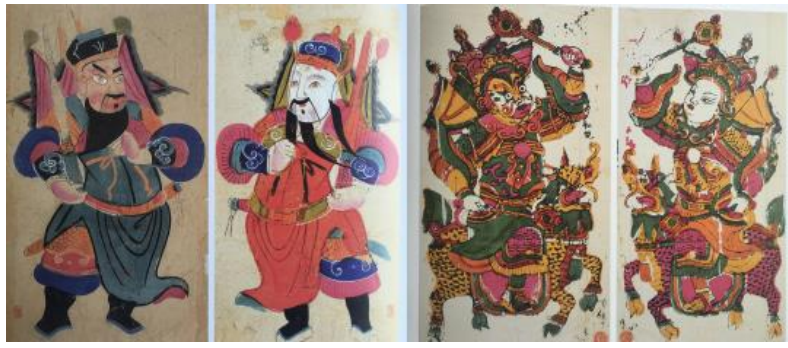

Fig. 12. The New Year pictures of Door-god in China.

4) The influence of the "Yin-Yang and Five Elements" concept of universe and life on the traditional patterns: In ancient times, the symbol of "swastikas" appeared in China, which symbolized the sun and fire and was then widely regarded as a symbol of auspiciousness. This diagram of the rotational structure of the sun comes from the ancient people's observation of natural things. The patterns that are based on the rotational structure are ubiquitous in the Chinese folk art. Here, through the relationship of the the unity of opposites, "Tai Ji Diagram" summarizes the origin, structure and properties of society, nature and human and gives a comprehensive expression of Chinese people's outlook on universe and life; this theoretical system is so large that it almost includes everything. The Shaanxi papercut work "The Snake Coiling the Rabbit" is made of red paper; the pattern of a snake is coiling an image of little rabbit in the center, which is popular in northern Shaanxi as a common theme for the folk wedding celebrations. There is a local proverb: "If you want to be rich, the snake should coil the rabbit". Originally, this represented the worship for the animal signs when a man and a woman are ready to get married. It was believed that the marriage of a man whose animal sign is snake and a woman whose animal sign is rabbit would bring good luck. Later, this pattern gradually evolved into a symbol of auspicious happiness and wealth. The rotational and dynamic snake and the static rabbit reflect a life state of the mutual growth, mutual infiltration, mutual influence and interdependence of Yin and Yang in "Fig. 13" and "Figure. 14".

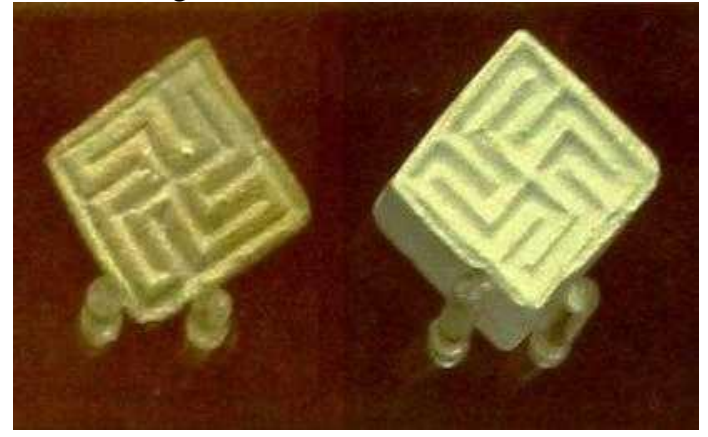

Fig. 13. The symbol of "swastikas"

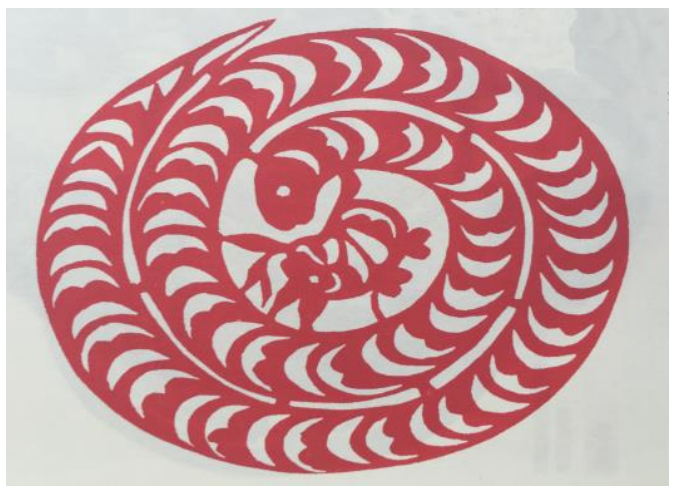

Fig. 14. The Shaanxi paper-cut work "The Snake Coiling the Rabbit"

\section{CONCLUSION}

As the basis of the traditional philosophy, the Chinese traditional view of Yin and Yang and outlook on life reflect people's desire to know the world and their worship for reproduction, that is, the human reproduction is continuous. This is a philosophical conclusion drawn by the ancestors of Chinese people through observing the human beings themselves and the universe --- they "based their judgment on themselves and the changes of the things around them"; this is also the basic cultural connotation of the national primitive art and the folk art. In a word, in the long history, 
the Yin-Yang philosophy has made people have a scientific understanding of society and nature and has become the theoretical basis for the Chinese folk art. The concept of YinYang and Five Elements has provided the theoretical support characterized by the unity of opposites for the traditional fine arts which is seeking for development; meanwhile, it makes the Chinese traditional art have unique features. People have further used the "concept of Yin and Yang" to explore the human world and to better understand the Chinese folk art. When people know these traditional philosophical concepts, they can use them to analyze the modern patterns and create more works on modern designs. In this way, the young people can enjoy, inherit and promote these philosophical ideas and the traditional culture.

\section{ACKNOWLEDGMENT}

This paper is funded by General Project of Art of The National Social Science Fund of China "Research on the development of cultural and creative products based on Museum rating criteria". (No.: 17BH160).

\section{REFERENCES}

[1] Zuo Hanzhong, The modeling of the Chinese folk arts[M], Changsha:Hunan Fine Arts Publishing House, 1998.

[2] Nan Caihong, The philosophical concept of Yin and Yang in the modeling of the Chinese folk arts [J], Qingdao: College of Fine Arts of Qingdao University of Technology.

[3] Zhang Yinghang, An Introduction to the Chinese traditional culture, Hangzhou: Zhejiang University Press, 2005.

[4] Luo Ming, Reflections on the relationship between the idea of "Yin and Yang" and the modern design art [J], Anhui: College of Communication of Anhui Normal University, 2009. 